T. Hosoh

Nagoya Math. J.

Vol. 59 (1975), 135-148

\title{
AMPLE VECTOR BUNDLES ON A RATIONAL SURFACE
}

\author{
TOSHIO HOSOH
}

\section{Introduction.}

On a complete non-singular curve defined over the complex number field $C$, a stable vector bundle is ample if and only if its degree is positive [3]. On a surface, the notion of the $H$-stability was introduced by F. Takemoto [8] (see $\S 1$ ). We have a simple numerical sufficient condition for an $H$-stable vector bundle on a surface $S$ defined over $\boldsymbol{C}$ to be ample; let $E$ be an $H$-stable vector bundle of rank 2 on $S$ with $\Delta(E)=c_{1}(E)^{2}-4 c_{2}(E) \geqq 0$, then $E$ is ample if and only if $c_{1}(E)>0$ and $c_{2}(E)>0$, provided $S$ is an abelian surface, a ruled surface or a hyperelliptic surface [9]. But the assumption above concerning $\Delta(E)$ evidently seems too strong. In this paper, we restrict ourselves to the projective plane $\boldsymbol{P}^{2}$ and a rational ruled surface $\Sigma_{n}$ defined over an algebraically closed field $k$ of arbitrary characteristic. We shall prove a finer assertion than that of [9] for an $H$-stable vector bundle of rank 2 to be ample (Theorem 1 and Theorem 3). Examples show that our result is best possible though it is not a necessary condition (see Remark (1) §2).

In $\S 1$, we shall recall the definition of $H$-stable vector bundles and their elementary properties proved by F. Takemoto [8].

In $\S 2$, we shall prove the following;

THEOREM 1. If $E$ is an $H$-stable vector bundle of rank 2 on $\boldsymbol{P}^{2}$ with $c_{1}(E) \geqq(-1 / 2) \Delta(E)$, then $E$ is ample.

In $\S 3$, we shall prove a similar sufficient condition for an $H$-stable vector bundle of rank 2 on $\Sigma_{n}$ to be ample (Theorem 3).

The author wishes to thank $H$. Umemura who called his attention to this problem and gave him many suggestions.

\section{§1. Preliminaries}

Let $k$ be an algebraically closed field of arbitrary characteristic.

Received August 30, 1974. 
Throughout this paper, the ground field $k$ will be fixed. Let $E$ be a vector bundle (i.e. a locally free sheaf) on a non-singular irreducible projective algebraic variety $X$ defined over $k$. We shall use the following notation;

$$
\begin{aligned}
h^{i}(X, E): & =\operatorname{dim}_{k} H^{i}(X, E) ; \text { the dimension of } H^{i}(X, E) . \\
E^{*}: & =\operatorname{Hom}_{O_{X}}\left(E, O_{X}\right) ; \text { the dual vector bundle of } E . \\
\chi(E) & :=\sum_{i}(-1)^{i} h^{i}(X, E) ; \text { the Euler-Poincaré characteristic of } E . \\
c_{i}(E) & ; \text { the } i \text {-th Chern class of } E .
\end{aligned}
$$

Let $H$ be an ample line bundle (i.e. invertible sheaf) on $X$ and $s=$ $\operatorname{dim} X$. We recall the deflnition of $H$-stable vector bundles [8].

DEFINITION. A vector bundle $E$ on $X$ is $H$-stable if for every nontrivial, non-torsion, quotient sheaf $F$ of $E, d(E, H) / r) E)<d(F, H) / r(F)$, where $d(F, H)=\left(c_{1}\left(F^{\prime}\right), H^{s-1}\right)$ with the intersection pairing (, ) and where $r(F)$ is the rank of $F$.

The following lemma is an immediate consequence of the definition.

LEMMA (1.1). (1) A vector bundle is H-stable if and only if it is $H^{\otimes n}$-stable for any natural number $n$.

(2) If $L$ is a line bundle, then $E$ is $H$-stable if and only if $E \otimes L$ is $H$-stable.

(3) If $E$ is $H$-stable and $d(E, H) \leqq 0$, then $H^{0}(X, E)=(0)$.

We say that a vector bundle $E$ is simple if any global endomorphism of $E$ is constant, i.e. $H^{0}(X$, End $(E))=k$. We know that an $H$-stable vector bundle is simple ([8] Corollary (1.8)). In the case of rank 2 vector bundles on $\boldsymbol{P}^{2}$, also the converse is true ([8] Proposition (4.1)), i.e. ;

LEMMA (1.2). Let $E$ be a vector bundle of rank 2 on $\boldsymbol{P}^{2}$, then the following conditions are equivalent

(1). $E$ is simple. (2). $E$ is $O^{P^{2}}(1)$-stable.

There is a very usefull criterion for a rank 2 vector bundle to be not simple ([7] Theorem 1.);

LEMMA (1.3). Let $E$ be a vector bundle of rank 2 on $X$, then the following conditions are equivalent.

(1). $E$ is not simple.

(2). There exists a line bundle $L$ on $X$ such that for $E^{\prime}=E \otimes L$, 
$h^{0}\left(X, E^{\prime}\right) \neq 0$ and $h^{0}\left(X, E^{*}\right) \neq 0$.

Let $E$ be a vector bundle on $X, P(E)$ the projective bundle on $X$ associated to $E$ and $O_{P(E)}(1)$ the tautological line bundle on $P(E)$ i.e. $\pi_{*}\left(O_{P(E)}(1)\right) \cong E, \pi$ being the natural projection of $P(E)$ onto $X$. If $L$ is a line bundle on $X$, then the line bundle $O_{P(E)}(1) \otimes \pi^{*}(L)$ is also the tautological line bundle on $\boldsymbol{P}(E \otimes L) \cong \boldsymbol{P}(E)$. If $M$ is a line bundle on $\boldsymbol{P}(E), M$ is isomorphic to a line bundle $O_{\boldsymbol{P}(E)}(1)^{\otimes n} \otimes \pi *(N)$ for some integer $n$ and some line bundle $N$ on $X$ (see EGA II. 4.1). A rational ruled surface is isomorphic to $\Sigma_{n}=\boldsymbol{P}\left(O_{P^{1}}(-n) \oplus O_{P^{1}}\right)$ for some nonnegative integer $n$. We denote the projection from $\Sigma_{n}$ to $P^{1}$ by $\pi_{n}$.

The following lemma plays an important role in the sequel.

LEMMA (1.4) Let $s$ be a section of the projection $\pi_{n}: \Sigma_{n} \rightarrow \boldsymbol{P}^{1}$, then;

(1) If the self-intersection number $(s, s)$ is non-positive, then $(s, s)$ $=-n$ and the direct image $\pi_{n^{*}}\left(O_{\Sigma_{n}}(s)\right)$ is isomorphic to the the vector bundle $O_{P_{1}}(-n) \oplus O_{P 1}$.

(2) If the self-intersection number $(s, s)$ is non-negative, then $(s, s)$ $\geqq n$ and the direct image $\pi_{n^{*}}\left(O_{\Sigma_{n}}(s)\right)$ is generated by its global sections.

Proof. We have an exact sequence on $\Sigma_{n}$;

$$
\left.0 \longrightarrow O_{\Sigma_{n}} \longrightarrow O_{\Sigma_{n}}(s) \longrightarrow O_{\Sigma_{n}}(s)\right|_{s} \longrightarrow 0
$$

Since $R^{1} \pi_{n^{*}}\left(O_{\Sigma_{n}}\right)=(0), \pi_{n^{*}}\left(O_{\Sigma_{n}}\right) \cong O_{P^{1}}, \pi_{n^{*}}\left(\left.O_{\Sigma_{n}}(s)\right|_{s}\right) \cong \boldsymbol{P}^{1}((s, s))$ and $\pi_{n^{*}}\left(O_{\Sigma_{n}}(s)\right)$ $\cong\left(O_{P_{1}}(-n) \oplus O_{P_{1}}\right) \otimes O_{P_{1}}(a)$ for some integer $a$, we have the following exact sequence;

$$
0 \longrightarrow O_{P^{1}} \longrightarrow\left(O_{P_{1}}(-n) \oplus O_{P_{1}}\right) \otimes O_{P^{1}}(a) \longrightarrow O_{P^{1}}((s, s)) \longrightarrow 0 \quad(*)
$$

(1) If $(s, s) \leqq 0$, then the exact sequence $(*)$ is split because $h^{1}\left(\boldsymbol{P}^{1}, O_{P^{1}}(t)\right)$ $=0$ for $t \geqq 0$. Hence we have;

$$
\left(O_{P^{1}}(-n) \oplus O_{P^{1}}\right) \otimes O_{P^{1}}(a) \cong O_{P 1}((s, s)) \oplus O_{P_{1}} .
$$

This is possible if and only if $a=0$ and $O_{P^{1}}((s, s)) \cong O_{P^{1}}(-n)$, hence $(s, s)=-n$ and $\pi_{n^{*}}\left(O_{\Sigma_{n}}(s)\right) \cong O_{P 1}(-n) \oplus O_{P_{1}}$.

(2) If $(s, s) \geqq 0$, then $O_{P 1}((s, s))$ is generated by its global sections. Hence we have that $\pi_{n^{*}}\left(O_{\Sigma_{n}}(s)\right)$ is generated by its global sections by virtue of the exact sequence $(*)$. This is possible if and only if $a-n \geqq 0$. On the other hand, $O_{P_{1}}((s, s))$ is isomorphic to $O_{P 1}(2 a-n)$ by $(*)$, which 
implies $(s, s)=2 a-n=2(a-n)+n \geqq n$.

The section on $\Sigma_{n}$ corresponding to the exact sequence;

$$
0 \longrightarrow O_{P_{1}} \longrightarrow O_{P^{1}}(-n) \oplus O_{P_{1}} \longrightarrow O_{P^{1}}(-n) \longrightarrow 0
$$

is called a minimal section of $\Sigma_{n}$ and denoted by $M$. Let $N$ be a fibre of $\pi_{n}$, then every divisor $D$ on $\Sigma_{n}$ is linearly equivalent to $a M+b N$ where $a=(D, N)$ and $b=(D, M)+a n$. A canonical divisor on $\Sigma_{n}$ is linearly equivalent to $-2 M-(n+2) N$.

\section{§ 2. Simple vector bundles on $\boldsymbol{P}^{2}$}

Let $E$ be a vector bundle of rank $r$ on $\boldsymbol{P}^{2}$ and $\ell$ be a line on $\boldsymbol{P}^{2}$, then the restriction $\left.E\right|_{\ell}$ of $E$ to $\ell$ is isomorphic to a direct sum of line bundles $L_{i}$ 's $(1 \leqq i \leqq r)$ [2]; we set;

$$
\alpha_{E}(\ell)=\min \left\{\operatorname{deg}\left(L_{i}\right) ; 1 \leqq i \leqq r\right\}
$$

Evidently the number $\alpha_{E}(\ell)$ is bounded above and below when $\ell$ runs through lines on $\boldsymbol{P}^{2}$. Hence we set;

$$
\begin{aligned}
& M(E):=\max \left\{\alpha_{E}(\ell) ; \ell \text { is a line on } \boldsymbol{P}^{2}\right\} \\
& m(E):=\min \left\{\alpha_{E}(\ell) ; \ell \text { is a line on } \boldsymbol{P}^{2}\right\}
\end{aligned}
$$

If $E$ is a vector bundle on $\boldsymbol{P}^{2}$, we put $E(n)=E \otimes O_{P^{2}}(1)^{\otimes n}$.

LEMMA (2.1) Let $E$ be a vector bundle on $\boldsymbol{P}^{2}$, then;

(1) If $M(E) \geqq-1$, then $h^{1}\left(\boldsymbol{P}^{2}, E(1)\right) \leqq h^{1}\left(\boldsymbol{P}^{2}, E\right)$.

(2) If $M(E) \geqq-1>m(E)$, then $h^{1}\left(\boldsymbol{P}^{2}, E(1)\right)<h^{1}\left(\boldsymbol{P}^{2}, E\right)$.

(3) If $M(E) \geqq-1$ and $h^{1}\left(P^{2}, E(1)\right)=h^{1}\left(P^{2}, E\right)$, then $E(1)$ is generated by its global sections.

Proof. (1) Let $\ell$ be a line with $\alpha_{E}(\ell)=M(E)$, then there is the following short exact sequence;

$$
O \longrightarrow O_{P^{2}}(-1) \longrightarrow O_{P^{2}} \longrightarrow O_{\ell} \longrightarrow 0
$$

Tensoring $E(1)$ with $(*)$, we get the short exact sequence;

$$
\left.0 \longrightarrow E \longrightarrow E(1) \longrightarrow E(1)\right|_{\ell} \longrightarrow 0
$$

and the long exact sequence of cohomologies;

$$
\cdots \longrightarrow H^{1}\left(\boldsymbol{P}^{2}, E\right) \longrightarrow H^{1}\left(\boldsymbol{P}^{2}, E(1)\right) \longrightarrow H^{1}\left(\ell,\left.E(1)\right|_{\ell}\right) \longrightarrow \cdots
$$

Since $\alpha_{E(1)}(\ell)=\alpha_{E}(\ell)+1 \geqq 0$, we have $h^{1}\left(\ell,\left.E(1)\right|_{\ell}\right)=0$, whence $h^{1}\left(\boldsymbol{P}^{2}, E(1)\right)$ 
$\leqq h^{1}\left(\boldsymbol{P}^{2}, E\right)$.

(2) By (1), we have $h^{1}\left(\boldsymbol{P}^{2}, E(1)\right) \leqq h^{1}\left(\boldsymbol{P}^{2}, E\right)$. Let $\ell$ be a line on $\boldsymbol{P}^{2}$ with $\alpha_{E}(\ell)=M(E)$, then as above we obtain the following long exact sequence of cohomologies;

$$
\begin{aligned}
\cdots & \longrightarrow H^{0}\left(\boldsymbol{P}^{2}, E(1)\right) \longrightarrow H^{0}\left(\ell,\left.E(1)\right|_{\ell}\right) \longrightarrow H^{1}\left(\boldsymbol{P}^{2}, E\right) \\
& \longrightarrow H^{1}\left(\boldsymbol{P}^{2}, E(1)\right) \longrightarrow H^{1}\left(\ell,\left.E(1)\right|_{\ell}\right)=(0) .
\end{aligned}
$$

If $h^{1}\left(\boldsymbol{P}^{2}, E(1)\right)=h^{1}\left(\boldsymbol{P}^{2}, E\right)$, then $H^{1}\left(\boldsymbol{P}^{2}, E\right) \cong H^{1}\left(\boldsymbol{P}^{2}, E(1)\right)$. Thus $\varphi: H^{0}\left(\boldsymbol{P}^{2}, E(1)\right)$ $\rightarrow H^{0}\left(\ell,\left.E(1)\right|_{\ell}\right)$ is surjective. By the way, let $\ell^{\prime}$ be a line on $\boldsymbol{P}^{2}$ with $\alpha_{E}\left(\ell^{\prime}\right)=m(E)$ and $x$ be the closed point of the intersection of $\ell$ and $\ell^{\prime}$, then $\psi: H^{0}\left(\ell,\left.E(1)\right|_{\ell}\right) \rightarrow E(1) \otimes k(x)$ is surjective since $\alpha_{E(1)}(\ell)=\alpha_{E}(\ell)+1$ $\geqq 0$. On the other hand $\psi^{\prime}: H^{0}\left(\ell^{\prime},\left.E(1)\right|_{\ell^{\prime}}\right) \rightarrow E(1) \otimes k(x)$ is not surjective because $\alpha_{E(1)}\left(\ell^{\prime}\right)=\alpha_{E}\left(\ell^{\prime}\right)+1 \leqq-1$. Furthermore we have the following commutative diagram;

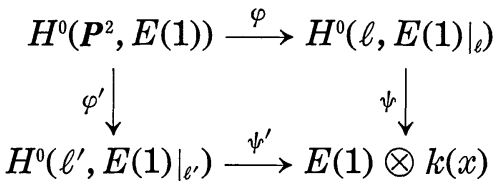

On the one hand, $\psi \circ \varphi$ is surjective because so are $\varphi$ and $\psi$. On the other hand, $\psi^{\prime} \circ \varphi^{\prime}$ is not surjective because not so is $\psi^{\prime}$. This is a contradiction.

(3) Let $x$ be any closed point of $\boldsymbol{P}^{2}$ and $\ell$ be a line passing through $x$. The assumptions $\alpha_{E}(\ell) \geqq m(E) \geqq-1$ and $h^{1}\left(\boldsymbol{P}^{2}, E(1)\right)=h^{1}\left(\boldsymbol{P}^{2}, E\right)$ imply that $H^{0}\left(\boldsymbol{P}^{2}, E(1)\right) \rightarrow H^{0}\left(\ell,\left.E(1)\right|_{\ell}\right)$ is surjective and $H^{1}\left(\ell,\left.E(1)\right|_{\ell}\right) \rightarrow E(1)$ $\otimes k(x)$ is surjective for any closed point $x$. By this and Nakayama's lemma $E(1)$ is generated by its global sections.

Let $X$ be a scheme defined over $k$ and $E_{1}, E_{2}$ vector bundles on $X$. If $E_{1}$ is ample and $E_{2}$ is generated by its global sections, then $E_{1} \otimes E_{2}$ is ample ([4] Corollary 1.9.). We get therefore the following proposition as a corollary to the above lemma.

Proposition (2.2) Let $E$ be a vector bundle on $\boldsymbol{P}^{2}$ with $M(E) \geqq-1$, then $E(a)$ is ample for any integer $a \geqq h^{1}\left(\boldsymbol{P}^{2}, E\right)+2$.

Proof. Put $b=h^{1}\left(\boldsymbol{P}^{2}, E\right)$, then by Lemma (2.1) we have;

$$
b=h^{1}\left(\boldsymbol{P}^{2}, E\right) \geqq h^{1}\left(\boldsymbol{P}^{2}, E(1)\right) \geqq \cdots \geqq h^{1}\left(\boldsymbol{P}^{2}, E(b)\right) \geqq 0 .
$$


Hence there must be an integer $c(0 \leqq c \leqq b)$ such that $h^{1}\left(\boldsymbol{P}^{1}, E(c)\right)=$ $h^{1}\left(\boldsymbol{P}^{2}, E(c+1)\right)$. By Lemma (2.1), $E(c+1)$ is generated by its global sections. Hence $E(a)$ is ample for any integer $a \geqq b+2$ because $O_{P^{2}}(n)$ is ample for any integer $n \geqq 1$.

For a vector bundle $E$ of rank 2 on a scheme we know that $E^{*} \cong E$ $\otimes(\operatorname{det} E)^{*}([6]$ Lemma 3.7). We shall use this fact in the next lemma.

If $E$ is a vector bundle on $\boldsymbol{P}^{2}$, we identify the Chern class $c_{i}(E)$ of $E$ with an integer by its degree.

LEMMA (2.3) Let $E$ be a simple vector bundle of rank 2 on $\boldsymbol{P}^{2}$, then;

(1) If $c_{1}(E) \leqq 0$, then $H^{0}\left(\boldsymbol{P}^{2}, E\right)=(0)$.

(2) If $c_{1}(E) \geqq-6$, then $H^{2}\left(\boldsymbol{P}^{2}, E\right)=(0)$.

Proof. We have $E^{*} \cong E \otimes(\operatorname{det} E)^{*} \cong E(c)$, where $c=-c_{1}(E)$. If $c_{1}(E) \leqq 0$, then $E$ can be regarded as a subsheaf of $E^{*}$. Hence $H^{0}\left(\boldsymbol{P}^{2}, E\right)$ $\subset H^{0}\left(\boldsymbol{P}^{2}, E^{*}\right)$. If $H^{0}\left(\boldsymbol{P}^{2}, E\right) \neq(0)$, then $H^{0}\left(\boldsymbol{P}^{2}, E^{*}\right) \neq(0)$. This contradicts to Lemma (1.3) and proves (1). The second assertion follows from (1) by the Serre duality.

Let $E$ be a vector bundle of rank 2 on a non-singular projective surface $S$. Define an integer $\Delta(E)$ to be $c_{1}(E)^{2}-4 c_{2}(E)$. It is easy to see that $-\Delta(E)$ is the second Chern class of End $(E)$. Hence, if $L$ is a line bundle on $S$, then $\Delta(E \otimes L)=\Delta(E)$. For given two integers $c_{1}$ and $c_{2}$, let $F\left(c_{1}, c_{2}\right)$ be the set of all simple vector bundles of rank 2 on $\boldsymbol{P}^{2}$ with $i$-th Chern class $c_{i}(i=1,2)$. Then $F\left(c_{1}, c_{2}\right)$ is not empty if and only if $c=c_{1}^{2}-4 c_{2}$ is negative and is not equal to $-4([6]$ Theorem 4.6). For a line bundle $L$ on $\boldsymbol{P}^{2}$, we put $F\left(c_{1}, c_{2}\right)(L)=\left\{E \otimes L ; E \in F\left(c_{1}, c_{2}\right)\right\}$. If $c_{1}$ is odd (resp. even), then for $L=O_{P_{2}}\left(-\left(c_{1}+1\right) / 2\right)\left(\operatorname{resp} . O_{P^{2}}\left(-c_{1} / 2\right)\right)$, $F\left(c_{1}, c_{2}\right)(L)=F(-1, n)$ (resp. $\left.F(0, m)\right)$ where $1-4 n=c_{1}^{2}-4 c_{2}$ (resp. $-4 m$ $\left.=c_{1}^{2}-4 c_{2}\right) . \quad F(-1, n)(\operatorname{resp} . F(0 ; m))$ is not empty if and only if $n \geqq 1$ (resp. $m \geqq 2$ ).

Now we can compute a lower bound of $m($ ) for simple vector bundles of rank 2 on $\boldsymbol{P}^{2}$ with fixed Chern classes.

Proposition (2.4) If $E$ is in $F(-1, n)$ (resp. $F(0, m)$ ), then;

$-n \leqq m(E) \leqq M(E) \leqq-1 \quad(\operatorname{resp} .-m+1 \leqq m(E) \leqq M(E) \leqq 0)$.

Proof. $M(E) \leqq-1$ (resp. $M(E) \leqq 0)$ is obvious, because $c_{1}(E)=-1$ (resp. $c_{1}(E)=0$ ). The Riemann-Roch theorem asserts that for a vector bundle $E^{\prime}$ of rank 2 on $\boldsymbol{P}^{2}$, 


$$
\chi\left(E^{\prime}\right)=2+\frac{3 c_{1}\left(E^{\prime}\right)}{2}+\frac{c_{2}\left(E^{\prime}\right)^{2}-2 c_{2}\left(E^{\prime}\right)}{2}
$$

Applying this to $E$ we have $\chi(E)=1-n$ (resp. $2-m$ ). On the other hand, by Lemma (2.3) $H^{0}\left(\boldsymbol{P}^{2}, E\right)=H^{2}\left(\boldsymbol{P}^{2}, E\right)=(0)$. Thus we obtain $h^{1}\left(\boldsymbol{P}^{2}, E\right)=n-1$ (resp. $\left.m-2\right)$. Let $\ell$ be any line on $\boldsymbol{P}^{2}$, then we have the following short exact sequence;

$$
\left.0 \longrightarrow E(-1) \longrightarrow E \longrightarrow E\right|_{\ell} \longrightarrow 0
$$

and the long exact sequence of cohomologies;

$$
\cdots \longrightarrow H^{1}\left(\boldsymbol{P}^{2}, E\right) \longrightarrow H^{1}\left(\ell,\left.E\right|_{\ell}\right) \longrightarrow H^{2}\left(\boldsymbol{P}^{2}, E(-1)\right) \longrightarrow \cdots
$$

Since $H^{2}\left(\boldsymbol{P}^{2}, E(-1)\right)=(0)$ by Lemma (2.3), we obtain $h^{1}\left(\ell,\left.E\right|_{\ell}\right) \leqq n-1$ (resp. $m-2$ ). Hence $\alpha_{E}(\ell) \geqq-n$ (resp. $-m+1$ ) for any line $\ell$.

LEMMA (2.5) Let $E$ be in $F(-1, n)$ (resp. $F(0, m)$ ). We put $b=$ $\min \left\{x ; H^{0}\left(\boldsymbol{P}^{2}, E(x)\right) \neq(0)\right\}$ (b is positive because $c_{1}(E(b))$ must be positive by Lemma (2.3)). Then $E(a)$ is ample for any integer $a \geqq n-b^{2}+b$ +1 (resp. $\left.m-b^{2}+1\right)$.

Proof. First we shall prove that $M(E(b)) \geqq 0$. Let $L$ be the tautological line bundle on $\boldsymbol{P}(E(b))$, then $H^{0}(\boldsymbol{P}(E(b)), L) \cong H^{0}\left(\boldsymbol{P}^{2}, E(b)\right) \neq(0)$. Take a member $D$ of the linear system $|L|$, then $\operatorname{Supp}(D)$ contains only a finite number of fibres of the projection $\pi: \boldsymbol{P}(E(b)) \rightarrow \boldsymbol{P}^{2}$. For if otherwise, there is an effective divisor $C$ on $\boldsymbol{P}^{2}$ such that $D-\pi^{-1}(C)$ $>0$, i.e. $H^{0}\left(\boldsymbol{P}(E(b)), L \otimes \pi^{*}\left(O_{\boldsymbol{P}^{2}}(-C)\right)\right) \neq(0)$. Meanwhile this is isomorphic to $H^{0}\left(\boldsymbol{P}^{2}, E(b) \otimes O_{P^{2}}(-C)\right)$. Thus by the definition of $b, C$ must be linearly equivalent to zero, which is not the case. Hence for a generic line $\ell$ on $\boldsymbol{P}^{2},\left.D\right|_{\pi-1(\ell)}$ is a section of the rational ruled surface $\pi^{-1}(\ell) \cong$ $\boldsymbol{P}\left(\left.E(b)\right|_{\ell}\right)$. On the otherhand, the self-intersection number $\left(\left.D\right|_{\pi-1(\ell)},\left.D\right|_{\pi-1(\ell)}\right)_{\pi-1(\ell)}$ $=c_{1}(E(b))>0$. Hence by Lemma (1.4), $\left.\left(\left.\pi\right|_{\ell}\right)_{*}\left(O_{\pi-1(\ell)}\left(\left.D\right|_{\pi-1(\ell)}\right)\right) \cong E(b)\right|_{\ell}$ is generated by its global sections. This shows that $M(E(b)) \geqq 0$.

The Chern classes of $E(b-1)$ are;

$$
\begin{aligned}
& c_{1}(E(b-1))=2 b-3 \quad(\text { resp. } 2 b-2) \\
& c_{2}(E(b-1))=b^{2}-3 b+2+n \quad\left(\text { resp. } b^{2}-2 b+1+m\right)
\end{aligned}
$$

By the Riemann-Roch theorem, we obtain;

$$
\chi(E(b-1))=b^{2}-n \quad\left(\text { resp. } b^{2}+b-m\right)
$$


On the other hand $H^{0}\left(\boldsymbol{P}^{2}, E(b-1)\right)=H^{2}\left(\boldsymbol{P}^{2}, E(b-1)\right)=(0)$. Hence we have $h^{1}\left(\boldsymbol{P}^{2}, E(b-1)\right)=n-b^{2}$ (resp. $\left.m-b^{2}-b\right)$.

Combining these results, by Proposition (2.2) $E(b-1)\left(a^{\prime}\right)$ is ample for any integer $a^{1} \geqq n-b^{2}+2$ (resp. $m-b^{2}-b+2$ ), i.e. $E(a)$ is ample for any integer $a \geqq n-b^{2}+b+1$ (resp. $\left.m-b^{2}+1\right)$.

CoROLlaRY (2.6) If $m(E)=-n$ (resp. $-m+1)$, then;

(1) $M(E) \geqq-1$.

(2) $h^{1}\left(\boldsymbol{P}^{2}, E(a)\right)=n-1-a$ (resp. $\left.m-2-a\right)$ for $0 \leqq a \leqq n-1$ (resp. $0 \leqq a \leqq m-2)$.

(3) For an integer a the following conditions are equivalent to each other ;

i) $E(a)$ is ample.

ii) $a \geqq n+1$ (resp. $m$ ).

iii) $c_{1}(E(a)) \geqq-(1 / 2) \Delta(E(a))$.

Proof. (3) ii) $\Leftrightarrow$ iii). $\quad c_{1}(E(a))=2 a-1 \quad$ (resp. $2 a$ ) and $\Delta(E(a))=$ $1-4 n$ (resp. $-4 m)$. Hence $c_{1}(E(a)) \geqq-(1 / 2) \Delta(E(a))$ if and only if $a \geqq n+1$ (resp. $m$ ).

ii) $\Rightarrow$ i). $\quad n+1 \geqq n-b^{2}+b+1 \quad$ (resp. $\left.m \geqq m-b^{2}+1\right)$ for any $b \geqq 1$. Hence $E(a)$ is ample by Lemma (2.5).

i) $\Rightarrow$ ii). If $E(a)$ is ample, then $m(E(a))=m(E)+a \geqq 1$. Hence $a \geqq-m(E)+1 \geqq n+1$ (resp. $m$ ).

(1) In the proof of (3), $b$ must be equal to 1 . Hence $M(E(1)) \geqq 0$ as we have shown in the proof of Lemma (2.5), i.e. $M(E) \geqq-1$.

(2) By the assumption $m(E)=-n$ (resp. $-m+1$ ) and (1), we have $M(E(a)) \geqq-1>m(E(a))$ for $0 \leqq a \leqq n-2$ (resp. $0 \leqq a \leqq m-3$ ). Hence by Lemma (2.1), we obtain;

$$
\begin{aligned}
& h^{1}\left(\boldsymbol{P}^{2}, E\right)>h^{1}\left(\boldsymbol{P}^{2}, E(1)\right)>\ldots>h^{1}\left(\boldsymbol{P}^{2}, E(n-1)\right) \\
& \quad\left(\text { resp. } h^{1}\left(\boldsymbol{P}^{2}, E\right)>h^{1}\left(\boldsymbol{P}^{2}, E(1)\right)>\ldots>h^{1}\left(\boldsymbol{P}^{2}, E(m-2)\right)\right) .
\end{aligned}
$$

Since $h^{1}\left(\boldsymbol{P}^{2}, E\right)=n-1$ (resp. $m-2$ ), this shows the assertion.

In the proof of Corollary (2.6) (3), we did not use the assumption $m(E)=-n$ (resp. $m(E)=-m+1)$ to show iii) $\Rightarrow \mathrm{i})$. Thus, we have proved the following;

THEOREM 1. If $E$ is a simple vector bundle of rank 2 on $\boldsymbol{P}^{2}$ with $c_{1}(E) \geqq-(1 / 2) \Delta(E)$, then $E$ is ample.

Remark (1) Theorem 1. is best possible in the following senses; 
i) For any integer $n \geqq 1$, there exists a simple vector bundle $E$ in $F(-1, n)$ such that $m(E)=-n$, i.e. $E(a)$ is ample if and only if $c_{1}(E(a))$ $\geqq-(1 / 2) \Delta(E(a)$ ) (see Corollary (2.6) (3)).

ii) For any integers $c_{1}$ and $c_{2}$, let $F^{\prime}\left(c_{1}, c_{2}\right)$ be the set of all vector bundles of rank 2 on $\boldsymbol{P}^{2}$ with its $i$-th Chern class being $c_{i}$, then $\inf \{m(E)$; $E$ in $\left.F^{\prime}\left(c_{1}, c_{2}\right)\right\}=-\infty$ i.e. for any integer $a$, there exists a vector bundle $E$ in $F^{\prime}\left(c_{1}, c_{2}\right)$ such that $m(E)<a$. Hence we can not drop the hypothesis "simple".

For the construction of examples satisfying i) or ii), see [6] Theorem 4.6, Theorem 3.13.

Remark (2) If $E$ is a simple vector bundle of rank 2 on $\boldsymbol{P}^{2}$ with $c_{1}(E) \geqq-(1 / 2) \Delta(E)$, then $E$ can be written in the form $E^{\prime} \otimes L$ where $E^{\prime}$ is generated by its global sections and $L$ is a very ample line bundle, hence if $k$ is the complex number field $C, E$ is positive in the sense of Griffiths [1].

\section{§ 3. $\boldsymbol{H}_{\alpha, \beta}$-stable vector bundles on a rational ruled surface.}

For a non-negative integer $n$, let $\Sigma_{n}$ be the rational ruled surface $\boldsymbol{P}\left(O_{P_{1}}(-n) \oplus O_{P_{1}}\right), M$ a minimal section on $\Sigma_{n}$ and $N$ a fibre of the projection $\pi_{n}: \Sigma_{n} \rightarrow \boldsymbol{P}^{1}$. Then every line bundle on $\Sigma_{n}$ is isomorphic to $O_{\Sigma_{n}}(a M+b N)$ for some integers $a$ and $b$. We denote the line bundle $O_{\Sigma_{n}}(a M+b N)$ by $L_{a, b}$.

LEMMA (3.1) (1) $L_{a, b}$ is ample if and only if $a$ is positive and $b$ - na is positive.

(2) $L_{a, b}$ is generated by its glebal sections if and only if a is nonnegative and $b-$ na is non-negative.

Proof. If $L_{a, b}$ is ample, then the intersection numbers $\left(L_{a, b}, N\right)=$ $a$ and $\left(L_{a, b}, M\right)=b-n a$ are positive by the Nakai criterion. Conversely if $a$ is positive and $b-n a$ is positive, then the self-intersection number $\left(L_{a, b}, L_{a, b}\right)=-a^{2} n+2 a b>-a^{2} n+2 a^{2} n=a^{2} n \geqq 0$. Any curve $C$ on $\Sigma_{n}$ is linearly equivalent to $a^{\prime} M+b^{\prime} N$ for some non-negative integers $a^{\prime}$ and $b^{\prime}$ such that $\left(a^{\prime}, b^{\prime}\right) \neq(0,0)$. Hence the intersection number $\left(L_{a, b}, C\right)=$ $a^{\prime}\left(L_{a, b}, M\right)+b^{\prime}\left(L_{a, b}, M\right)=a^{\prime}(-n a+b)+b^{\prime} a$ is positive. Therefore $L_{a, b}$ is ample by the Nakai criterion.

(2) If $L_{a, b}$ is generated by its global sections then the tensor product $L_{a, b} \otimes L_{1, n+1}=L_{a+1, b+n+1}$ is ample since $L_{1, n+1}$ is ample by (1). Hence 
$a+1$ is positive and $-n(a+1)+b+n+1$ is positive i.e. $a$ and $b-n a$ are non-negative. Conversely if $a$ and $b-n a$ are non-negative, then $L_{a, b}$ is generated by its global sections. In fact, $L_{1, n}$ is generated by its global sections and $L_{0,1}$ is so. Hence $L_{a, b}=L_{1, n}^{\otimes a} \otimes L_{0,1}^{\otimes(b-n a)}$ is generated by its global sections.

We denote the divisor $\alpha(M+n N)+\beta N$ by $H_{\alpha, \beta}$. Then the intersection numbers $\left(H_{\alpha, \beta}, N\right)$ and $\left(H_{\alpha, \beta}, M\right)$ are $\alpha$ and $\beta$ respectively and Lemma (3.1) (1) is restated as follows; $H_{\alpha, \beta}$ is ample if and only if $\alpha>0$ and $\beta>0$. We also denote $H_{1,1}=M+(n+1) N$ by $H$, then $H$ is very ample and any irreducible member of the linear system $|H|$ is isomorphic to the projective line $\boldsymbol{P}^{1}$. Let $E$ be a vector bundle of rank $r$ on $\Sigma_{n}$ and $\ell$ be an irreducible member of the linear system $|H|$, then the restriction $\left.E\right|_{\ell}$ of $E$ to $\ell$ is isomorphic to direct sum $L_{1} \oplus \cdots \oplus L_{r}$ of line bundles $L_{i}$ 's on $\ell$. We set;

$$
\alpha_{E}(\ell):=\min \left\{\operatorname{deg} L_{i} ; 1 \leqq i \leqq r\right\}
$$

and

$$
\begin{aligned}
& M(E)=\max \left\{\alpha_{E}(\ell) ; \ell \text { is an irreducible member of }|H|\right\} \\
& m(E)=\min \left\{\alpha_{E}(\ell) ; \ell \text { is an irreducible member of }|H|\right\}
\end{aligned}
$$

If $E$ is a vector bundle on $\Sigma_{n}$ and $D$ is a divisor on $\Sigma_{n}$, we put $E(D)=E \otimes O_{\Sigma_{n}}(D)$.

Lemma (3.2) Let $E$ be a vector bundle on $\Sigma_{n}$ then;

(1) If $M(E) \geqq-n-2$, then $h^{1}\left(\Sigma_{n}, E\right) \geqq h^{1}\left(\Sigma_{n}, E(H)\right.$ ).

(2) If $M(E) \geqq-n-2>m(E)$, then $h^{1}\left(\Sigma_{n}, E\right)>h^{1}\left(\Sigma_{n}, E(H)\right)$.

(3) If $m(E) \geqq-n-2$ and $h^{1}\left(\Sigma_{n}, E\right)=h^{1}\left(\Sigma_{n}, E(H)\right)$, then $E(H)$ is generated by its global sections.

Proof. The self-intersection number $(H, H)$ is $n+2$, so the proof is similar to that of Lemma (2.1). Hence we omit it.

The following proposition can be proved as a corollary to Lemma (3.2) and the proof is similar to that of Proposition (2.2).

Proposition (3.3) If $E$ is a vector bundle on $\Sigma_{n}$ with $M(E) \geqq-n$ -2 , then $E(a H)$ is ample for any integer $a \geqq h^{1}\left(\Sigma_{n}, E\right)+2$.

For any integers $a, b$ and $c$, we set; 


$$
\begin{aligned}
F_{n}(a, b ; c):= & \{E ; E \text { is a simple vector bundle of rank } 2 \text { on } \\
& \left.\Sigma_{n} \text { with } c_{1}(E)=a M+b N \text { and } c_{2}(E)=c\right\}
\end{aligned}
$$

If $L$ is a line bundle on $\Sigma_{n}$, we also set;

$$
F_{n}(a, b ; c)(L):=\left\{E \otimes L ; E \text { is in } F_{n}(a, b ; c)\right\}
$$

Then for any integers $a, b$ and $c$ there exists a line bundle $L$ on $\Sigma_{n}$ such that;

(1) If $a$ is even and $b$ is even $F_{n}(a, b ; c)(L)=F_{n}(0,0 ; r)$ where $-4 r=-a^{2} n+2 a b-4 c$.

(2) If $a$ is even and $b$ is odd $F_{n}(a, b ; c)(L)=F_{n}(0,-1 ; r)$ where $-4 r=-a^{2} n+2 a b-4 c$.

(3) If $a$ is odd and $b$ is even $F_{n}(a, b ; c)(L)=F_{n}(-1,0 ; r)$ where $-n-4 r=-a^{2} n+2 a b-4 c$.

(4) If $a$ is odd and $b$ is odd $F_{n}(a, b ; c)(L)=F_{n}(-1,-1 ; r)$ where $-n+2-4 r=-a^{2} n+2 a b-4 c$.

M. Maruyama ([6] Theorem 4.15) proved that;

(1) $F_{n}(0,0 ; r)$ is not empty if and only if $r \geqq 2$.

(2) $F_{n}(0,-1 ; r)$ is not empty if and only if $r \geqq 1$.

(3) $F_{n}(-1,0 ; r)$ is not empty if and only if $r \geqq 1$.

(4) $F_{n}(-1,-1 ; r)$ is not empty if and only if $r \geqq 1$ when $n \neq 0$, $r \geqq 2$ when $n=0$.

LEMMA (3.4) Let $E$ be a simple vector bundle of rank 2 on $\Sigma_{n}$ with $c_{1}(E)=a M+b N$, then

(1) If $a \leqq 0$ and $b \leqq 0$, then $H^{\circ}\left(\Sigma_{n}, E\right)=(0)$.

(2) If $a \geqq-4$ and $b \geqq-2(n+2)$, then $H^{2}\left(\Sigma_{n}, E\right)=(0)$.

Proof. The canonical line bundle on $\Sigma_{n}$ is isomorphic to the line bundle $L_{-2,-n-2}$, so the proof is similar to that of Lemma (2.3).

We say that a set $S$ of vector bundles on a $k$-scheme $X$ is bounded if there exists an algebraic $k$-scheme $T$ and a vector bundle $V$ on $T \times X$ such that each $E$ in $S$ is isomorphic to $V_{t}=\left.V\right|_{t \times X}$ for some closed point $t$ in $T$.

THEOREM 2. For any integers $a, b$ and $c, F_{n}(a, b ; c)$ is bounded.

Proof. It is sufficient to prove the theorem for $-1 \leqq a, b \leqq 0$. 
We shall prove the theorem for $F_{n}(0,0 ; r)$ only, since the other cases are similar. By a theorem of Kleiman ([5] Theorem 1.13), it is sufficient to show that there are integers $m_{1}$ and $m_{2}$ such that for any $E$ in $F_{n}(0,0 ; r)$, i) $h^{0}\left(\Sigma_{n}, E\right) \leqq m_{1}$ and ii) $h^{0}\left(\ell,\left.E\right|_{\ell}\right) \leqq m_{2}$ for a generic member $\ell$ of the linear system $|H|$. By Lemma $(3.4), h^{0}\left(\Sigma_{n}, E\right)=0$ for any $E$ in $F_{n}(0,0 ; r)$. We now show ii). The Riemann-Roch theorem asserts that for a vector bundle $E^{\prime}$ of rank 2 on $\Sigma_{n}$,

$$
\chi\left(E^{\prime}\right)=2+\frac{\left(2 M+(n+2) N, c_{1}\left(E^{\prime}\right)\right)}{2}+\frac{c_{1}\left(E^{\prime}\right)^{2}-2 c_{2}\left(E^{\prime}\right)}{2} .
$$

Applying this to $E$ in $F_{n}(0,0 ; r)$, we have $\chi(E)=2-r$. On the other hand, by Lemma (3.4), $h^{0}\left(\Sigma_{n}, E\right)=h^{2}\left(\Sigma_{n}, E\right)=0$. Thus we obtain $h^{1}\left(\Sigma_{n}, E\right)=r-2$. Let $\ell$ be a generic member of the linear system $|H|$, then we have the following short exact sequence;

$$
\left.0 \longrightarrow E(-H) \longrightarrow E \longrightarrow E\right|_{\ell} \longrightarrow 0
$$

and the long exact sequence of cohomologies;

$$
\cdots \longrightarrow H^{1}\left(\Sigma_{n}, E\right) \longrightarrow H^{1}\left(\ell,\left.E\right|_{\ell}\right) \longrightarrow H^{2}\left(\Sigma_{n}, E(-H)\right) \longrightarrow \cdots
$$

Since $c_{1}(E(-H))=-2 M-2(n+1) N, h^{2}\left(\Sigma_{n}, E(-H)\right)=0$ by Lemma (3.4). Hence we obtain;

$$
h^{1}\left(\ell,\left.E\right|_{\ell}\right) \leqq r-2 .
$$

On the other hand, by the Riemann-Roch theorem for a vector bundle of rank 2 on the projective line, we have;

$$
h^{0}\left(\ell,\left.E\right|_{\ell}\right)-h^{1}\left(\ell,\left.E\right|_{\ell}\right)=2+\operatorname{deg}\left(c_{1}\left(\left.E\right|_{\ell}\right)\right)=2 .
$$

Hence we obtain $h^{0}\left(\ell,\left.E\right|_{\ell}\right) \leqq r$.

LEMMA (3.5) Let $E$ be a simple vector bundle of rank 2 on $\Sigma_{n}$ with $c_{1}(E)=a M+b N$ such that $-1 \leqq a, b \leqq 0$. Put $d=\min \left\{x ; h^{0}\left(\Sigma_{n}, E(x H)\right)\right.$ $\neq 0\}$ ( $d$ is positive by Lemma (3.4)). If there exist integers $\alpha$ and $\beta$ with $\alpha \geqq 1, \beta \geqq 1$ and $1 / 2 \leqq \beta / \alpha \leqq n+3$ if $n \neq 0,1 / 3 \leqq \beta / \alpha \leqq 3$ if $n=0$ such that $E$ is $H_{\alpha, \beta}$ stable, then $M(E(d H)) \geqq 0$.

Proof. We shall prove the theorem for $a=0$ and $b=0$ only since the other cases are similar. Let $X$ be the projective bundle $\boldsymbol{P}(E(d H))$ on $\Sigma_{n}, \pi: X \rightarrow \Sigma_{n}$ the projection and $L$ the tautological line bundle on $X$. Let $D^{\prime}$ be a member of the linear system $|L|$ on $X$, then $D^{\prime}$ can be 
written in the form $D^{\prime}=D+\pi^{-1}(C)$ where $D$ is an irreducible divisor on $X$ and $C$ is an effective divisor on $\Sigma_{n}$ i.e. $C$ is linearly equivalent to $x M+y N(x \geqq 0, y \geqq 0)$. Put $E^{\prime}=\pi_{*}\left(O_{X}(D)\right) \cong E(d H-x M-y N)$. Let $\ell$ be a generic member of the linear system $|H|$ on $\Sigma_{n}$, then $\left.D\right|_{\pi^{-1}(\ell)}$ is a section of the rational ruled surface $\pi^{-1}(\ell)$ and the self-intersection number $\left(\left.D\right|_{\pi^{-1(\ell)}},\left.D\right|_{\pi^{-1(\ell)}}\right)_{\pi^{-1}(\ell)}=\left(c_{1}(E(d H-x M-y N)), H\right)=2 d(n+2)-$ $2(x+y)$. If $2 d(n+2)-2(x+y) \geqq 0$, then $\alpha_{E^{\prime}}(\ell) \geqq 0$ by Lemma (1.4). Hence $\alpha_{E(d H)}(\ell)=\alpha_{E^{\prime}}(\ell)+x+y \geqq 0$, therefore $M(E(d H)) \geqq 0$. If $2 d(n+2)$ $-2(x+y)<0$, then $\alpha_{E \prime}(\ell)=2 d(n+2)-2(x+y)$ by Lemma (1.4). Hence $\alpha_{E(d H)}(l)=2 d(n+2)-(x+y)$. We shall show that $2 d(n+2) \geqq$ $x+y$. Now assume that $2 d(n+2)<x+y$, then we shall show a contradiction. Since $h^{0}\left(\Sigma_{n}, E^{\prime}\right) \neq 0$ and $E^{\prime}$ is $H_{\alpha, \beta}$-stable, $\left(c_{1}\left(E^{\prime}\right), H_{\alpha, \beta}\right)=$ $2 \beta(d-x)+2 \alpha(d(n+1)-y)>0$ by Lemma (1.1), hence $\beta d+\alpha d(n+1)$ $>\beta x+\alpha y$. We shall consider two cases i) $\beta \leqq \alpha$ and ii) $\beta \geqq \alpha$ separately.

i) Assume that $\beta \leqq \alpha$. If $n \neq 0$, then $\beta d+\alpha d(n+1) \leqq \alpha d(n+2)$ and $\beta x+\alpha y \geqq \beta(x+y)$, hence $\alpha d(n+2)>\beta(x+y)>2 \beta d(n+2)$. This contradicts to $1 / 2 \leqq \beta / \alpha$. If $n=0$, then $3 \beta \geqq \alpha$. Hence $\beta d+\alpha d \leqq 4 \beta d$ and $\beta x+\alpha y \geqq \beta(x+y)>4 \beta d$, therefore $4 \beta d>4 \beta d$. This is a contradiction.

ii) Assume that $\beta \geqq \alpha$. Then $\beta d+\alpha d(n+1) \leqq \alpha d(n+3)+\alpha d(n+1)$ $=2 \alpha d(n+2)$, and $\beta x+\alpha y \geqq \alpha(x+y)>2 \alpha d(n+2)$. Hence $2 \alpha d(n+2)$ $>2 \alpha d(n+2)$, this is a contradiction.

For any integers $a, b$ and $c$, we set; $F_{n}^{0}(a, b ; c):=\left\{E\right.$ in $F_{n}(a, b ; c) ; E$ is $H_{\alpha, \beta}$-stable for some $\alpha$ and $\beta$ with $1 / 2 \leqq \beta / \alpha \leqq n+3$ if $n \neq 0,1 / 3 \leqq \beta / \alpha \leqq 3$ if $n=0\}$

Corollary (3.6) (1) If $E$ is in $F_{n}^{0}(0,0 ; r)$ then $E(r H)$ is ample.

(2) If $E$ is in $F_{n}^{0}(0,-1 ; r)$ then $E((r+1) H)$ is ample.

(3) If $E$ is in $F_{n}^{0}(-1,0 ; r)$ then $E((r+1) H)$ is ample.

(4) If $E$ is in $F_{n}^{0}(-1,-1 ; r)$ then $E((r+1) H)$ is ample.

Proof. The proof is similar to that of Corollary (2.6), so we omit it.

THEOREM 3. Let $E$ be a simple vector bundle of rank 2 on $\Sigma_{n}$ with $c_{1}(E)=a M+b N$. Assume that $E$ is $H_{\alpha, \beta}$-stable for some $\alpha \geqq 1$ and $\beta \geqq 1$ such that $1 / 2 \leqq \beta / \alpha \leqq n+3$ if $n \neq 0,1 / 3 \leqq \beta / \alpha \leqq 3$ if $n=0$, then the intersection numbers $\left(c_{1}(E), N\right)=a,\left(c_{2}(E), M\right)=b-n a$ and;

(1) If $a$ is even, $b$ is even and $a \geqq 2 r, b-n a \geqq 2 r$ where $-4 r=$ 
$\Delta(E)$, then $E$ is ample.

(2) If $a$ is even, $b$ is odd and $a \geqq 2(r+1), b-n a \geqq 2(r+1)-1$ where $-4 r=\Delta(E)$, then $E$ is ampe.

(3) If $a$ is odd, $b$ is even and $a \geqq 2(r+1)-1, b-n a \geqq 2(r+1)$ $+n$ where $-n-4 r=\Delta(E)$, then $E$ is ample.

(4) If $a$ is odd, $b$ is odd and $a \geqq 2(r+1)-1, b-n a \geqq 2(r+1)$ $+n-1$ where $-n+2-4 r=\Delta(E)$, then $E$ is ample.

Proof. We shall prove the case (1) only since the other cases are similar. Let $E$ be an $H_{\alpha, \beta}$-stable vector bundle of rank 2 which satisfies the conditions of (1), then $E$ is written in the form $E^{\prime}(r H) \otimes L_{a^{\prime}, b^{\prime}}$ where $E^{\prime}$ is in $F_{n}^{0}(0,0 ; r)$ and $a^{\prime}=a / 2-r, b^{\prime}=b / 2-r(n+1) . \quad E^{\prime}(r H)$ is ample by Corollary (3.6) and $L_{a^{\prime}, b^{\prime}}$ is generated by its global sections by Lemma (3.1) because $a^{\prime}=a / 2-r \geqq 0$ and $b^{\prime}-n a^{\prime}=b / 2-r(n+1)-$ $n(a / 2-r)=1 / 2(b-n a-2 r) \geqq 0$, therefore $E=E^{\prime}(r H) \otimes L_{a^{\prime}, b^{\prime}}$ is ample.

\section{REFERENCES}

[1] Griffiths, P., Hermitian differential geometry, Chern classes, and positive vector bundles, Global Analysis, papers in honor of $\mathrm{K}$. Kodaira, Univ. of Tokyo press (1969) 185-251.

[2] Grothendieck, A., Sur la classification des fibrés holomorphes sur la sphére de Riemann, Amer. J. Math., 79 (1957) 121-138.

[3] Hartshorne, R., Ample vector bundles on curves, Nagoya Math. J., 43 (1971) 73-89.

[4] —- Ample subvarieties of algebraic varieties, Lecture notes in Math., Springer, 156 (1970).

[ 5 ] Kleiman, S., Les theoremes de finitude pour le foncteur de Picard, SGA 6, exposé 13.

[6] Maruyama, M., On a family of algebraic vector bundles, Number Theory, Algebraic Geometry and Commutative Algebra, in honor of Y. Akizuki, Kinokuniya, Tokyo, (1973) 95-146.

[7] Schwarzenberger, R. E. L., Vector bundles on algebraic surfaces, Proc. London Math. Soc., (3) 11 (1961) 601-622.

[8] Takemoto, F., Stable vector bundles on algebraic surfaces, Nagoya Math. J., 47 (1972) 29-48.

[9] Umemura, H., Some results in the theory of vector bundles, Nagoya Math. J., 52 (1973) 97-128.

Nagoya University 